\title{
In vitro metabolism of pyripyropene A and ACAT inhibitory activity of its metabolites
}

\author{
Daisuke Matsuda ${ }^{1}$, Taichi Ohshiro ${ }^{1,2}$, Masaki Ohtawa ${ }^{1}$, Hiroyuki Yamazaki ${ }^{1}$, Tohru Nagamitsu ${ }^{1}$ \\ and Hiroshi Tomoda ${ }^{1}$
}

Pyripyropene A (PPPA, 1) of fungal origin, a selective inhibitor of acyl-CoA:cholesterol acyltransferase 2 (ACAT2), proved orally active in atherogenic mouse models. The in vitro metabolites of 1 in liver microsomes and plasma of human, rabbit, rat and mouse were analyzed by ultra fast liquid chromatography and liquid chromatography/tandem mass spectrometry. In the liver microsomes from all species, successive hydrolysis occurred at the 1-O-acetyl residue, then at the 11- 0 -acetyl residue of 1 , while the 7-0-acetyl residue was resistant to hydrolysis. Furthermore, dehydrogenation of the newly generated 11-alcoholic hydroxyl residue occurred in human and mouse-liver microsomes, while oxidation of the pyridine ring occurred in human and rabbit liver microsomes. On the other hand, hydrolysis of the 7-O-acetyl residue proceeded only in the mouse plasma. These data indicated that the in vitro metabolic profiles of 1 have subtle differences among animal species. All of the PPPA metabolites observed in liver microsomes and plasma markedly decreased ACAT2 inhibitory activity. These findings will help us to synthesize new PPPA derivatives more effective in in vivo study than 1.

The Journal of Antibiotics (2015) 68, 27-34; doi:10.1038/ja.2014.91; published online 9 July 2014

\section{INTRODUCTION}

Acyl-CoA:cholesterol acyltransferase (ACAT, EC 2.3.1.26) is a promising therapeutic target for the treatment or prevention of hypercholesterolemia and atherosclerosis. ${ }^{1-3}$ Although a large number of synthetic ACAT inhibitors were developed in 1980s and 1990s, they caused numerous side effects including diarrhea and adrenal gland toxicity. ${ }^{4-6}$ Interestingly, two ACAT isozymes (ACAT1 and ACAT2) with distinct patterns of expression in tissues and physiological functions were discovered in the late $1990 \mathrm{~s}^{7-11}$ In parallel with the findings, non-selective ACAT inhibitors, avasimibe and pactimibe, which inhibit both ACAT1 and ACA2, were developed. Unfortunately, they did not appear to limit the progression of atherosclerosis in clinical trials. ${ }^{12-14}$

Pyripyropene A (PPPA, 1, Figure 1), isolated from the culture broth of Aspergillus fumigatus FO-1289, ${ }^{15-17}$ was found to be the first inhibitor highly selective toward ACAT2 isozyme. ${ }^{18-20}$ Among approximately 200 PPPA derivatives we had prepared in 1990s (the first generation derivatives), ${ }^{21-23} 1$ showed the highest selectivity toward ACAT2. ${ }^{24}$ Accordingly, $\mathbf{1}$ was chosen as the best candidate for investigating whether ACAT2 selective inhibition exerts in vivo atheroprotective activity. The in vivo experiments appeared worthy because no in vivo efficacy had been tested using ACAT2 selective inhibitors. As a result, we demonstrated that $\mathbf{1}$ was orally active in in vivo mouse studies, reducing total cholesterol levels in plasma and atherosclerotic lesion area in mouse models without causing side effects. $^{25}$
As a next step for further development, synthesis of new PPPA derivatives with more potent and more selective activity against ACAT2 than 1 was required. For this purpose, potential in vitro metabolism of $\mathbf{1}$ in plasmas and liver microsomes from various animal samples including mice and humans was investigated.

In this study, PPPA metabolites were identified by ultra fast liquid chromatography (UFLC) and liquid chromatography/tandem mass spectrometry (LC-MS) in comparison with the authentic derivatives chemically semi-synthesized. Finally, potential metabolic pathways of $\mathbf{1}$ in the liver and plasma were deduced. Furthermore, ACAT inhibitory activity of the metabolites was evaluated in our established cell-based assay.

\section{RESULTS}

Synthesis and UFLC analysis of PPPA derivatives

Esters, amides and thioester residues could be hydrolyzed by carboxyesterase, amide hydrolase and thioesterase, respectively, which show ubiquitous expression with high levels in various tissues, ${ }^{26}$ so that potential metabolites of $\mathbf{1}$ can be deduced. Therefore, we synthesized possible PPPA derivatives; 7-deacetyl PPPA (3), 1,7,11trideacetyl PPPA (8) and $N$-oxidized PPPA (10). Furthermore, as shown in Scheme 1, other novel PPPA derivatives 2, 4, 6 and 9 were synthesized. 1,7,11-Trideacetyl PPPA (8) was treated with $(t \mathrm{Bu})_{2-}$ $\mathrm{Si}(\mathrm{OTf})_{2}$ followed by acetylation at 7-hydroxy group to afford $\mathbf{1 2}$. Regioselective mono-deprotection of $\mathbf{1 2}$ in the presence of $\mathrm{NH}_{4} \mathrm{~F}$ afforded 13 as the mono-deprotected 14 in $78 \%$ yield along with

${ }^{1}$ Graduate School of Pharmaceutical Sciences, Kitasato University, Tokyo, Japan and ${ }^{2}$ Section on Lipid Sciences, Department of Pathology, Wake Forest School of Medicine, Winston-Salem, NC, USA

Correspondence: Professor H Tomoda, Graduate School of Pharmaceutical Sciences, Kitasato University, 5-9-1 Shirokane, Minato-ku, Tokyo 108-8641, Japan. E-mail: tomodah@pharm.kitasato-u.ac.jp

Received 9 April 2014; revised 12 May 2014; accepted 10 June 2014; published online 9 July 2014 
small amounts of regioisomer 14 (5.0\%) and 1,11-dideacetyl PPPA (6) (5.0\%). TEMPO (2,2,6,6-tetramethylpiperidine 1-oxyl)-mediated regioselective oxidation of $\mathbf{1 3}$ followed by desilylation at 1-position gave 9 in $47 \%$ yield from 13. An acetylation/deprotection sequences of 13 and 14 afforded 1-deacetyl PPPA (2) and 11-deacetyl PPPA (4) in $90 \%$ and $84 \%$ yield (two steps), respectively. Furthermore, synthesis

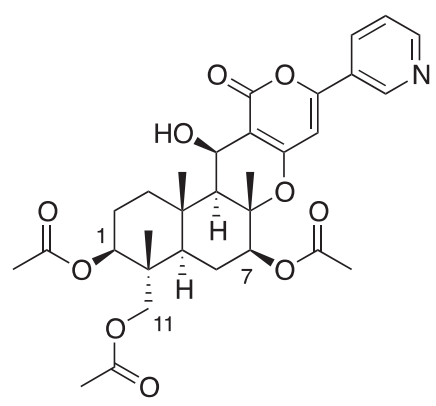

Pyripyropene A (PPPA,1)

Figure 1 The structure of pyripyropene $\mathrm{A}$. of the dideacetyl PPPA derivatives 5 and 7 was carried out as illustrated in Scheme 2. Protection of the 7-hydroxyl group of 11 by allyloxycarbonyl (alloc) group followed by the regioselective monodeprotection of silylene acetal $\mathbf{1 6}$ gave as a major product along with a small account of regioisomer 17. Finally, acetylation and deprotection of silylether and alloc group of $\mathbf{1 6}$ and $\mathbf{1 7}$ afforded 1,7-dideacetyl PPPA (5) and 7,11-dideacetyl PPPA (7) in three steps, respectively. Details of spectral data of PPPA derivatives are available from the Supporting Data.

Next, synthetic PPPA derivatives were analyzed by UFLC and LCMS with an octadecylsilane column. Under the routine conditions, 1 to 10 were eluted at the respective retention times of $3.8,3.1,3.0,3.5$, $2.2,2.8,2.6,1.9,2.9$ and $3.6 \mathrm{~min}$ by UFLC and of $11.5\left([\mathrm{M}+\mathrm{H}]^{+}\right.$ 584), 8.6 (542), 7.4 (542), 10.6 (542), 3.3 (500), 5.9 (500), 4.7 (500), 2.7 (458), 7.3 (498) and $10.5 \mathrm{~min}(600)$ by LC-MS (Table 1).

In vitro PPPA metabolism in human-, rabbit-, rat- and mouse-liver microsomes

PPPA (1) was incubated with human-, rabbit-, rat- and mouse-liver microsomes in the NADPH-generating system for various incubation periods. Without liver microsomes, 1 was stable and no PPPA

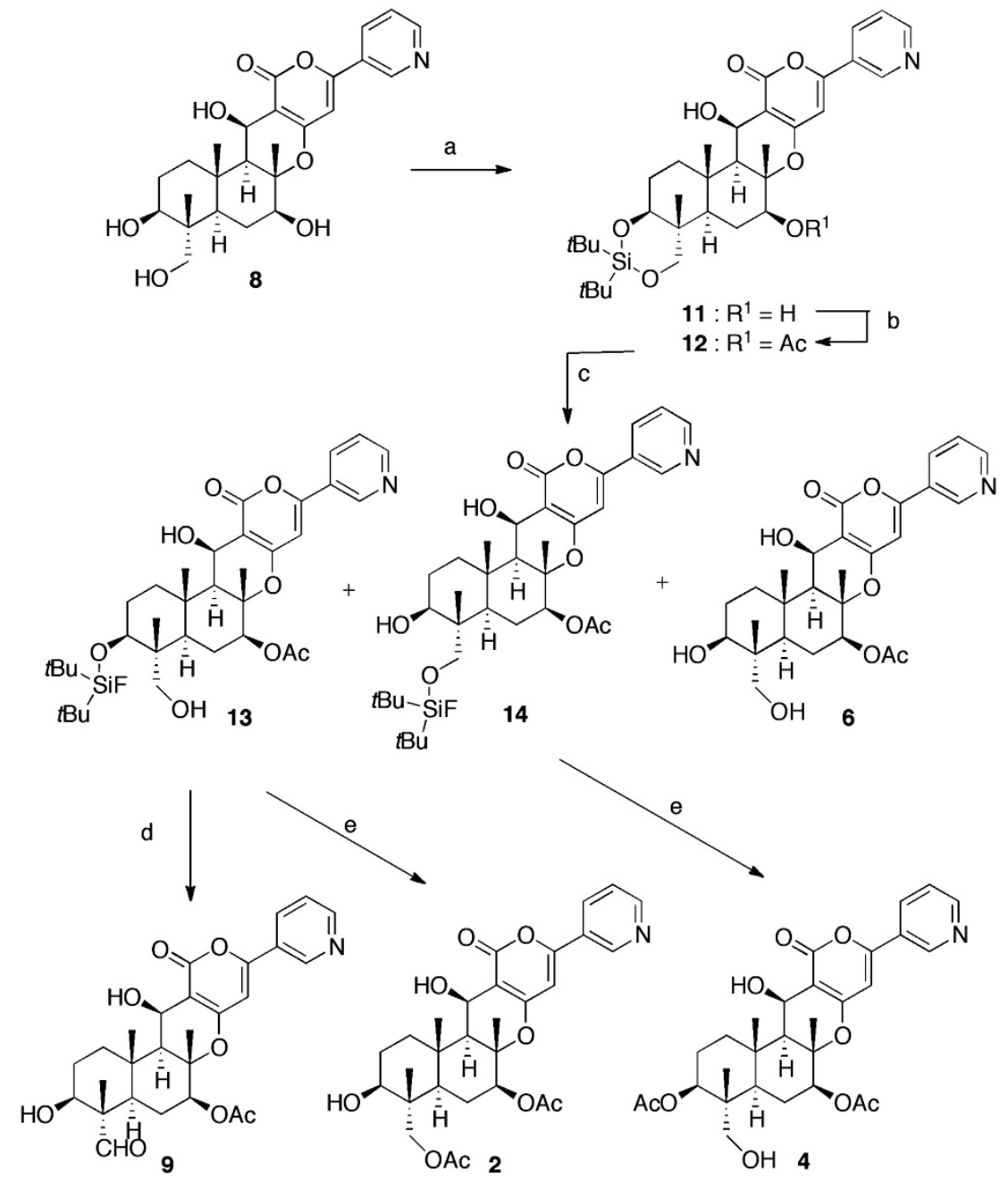

Scheme 1 Synthesis of novel pyripyropene A derivatives 2, 4, 6 and 9. Reagents and conditions: (a) (tBu $)_{2} \mathrm{Si}(\mathrm{OTf})_{2}$, 2,6-lutidine, DMF (dimethylformamide), r.t., $0.5 \mathrm{~h}$, quant.; (b) $\mathrm{Ac}_{2} \mathrm{O}, \mathrm{Et}_{3} \mathrm{~N}, \mathrm{DMAP}, \mathrm{CH}_{2} \mathrm{Cl}_{2}, \mathrm{O}^{\circ} \mathrm{C}, 1 \mathrm{~h}$, quant.; (c) $\mathrm{NH}_{4} \mathrm{~F}, \mathrm{MeOH}$, r.t., $3 \mathrm{~h}, 13: 78 \%, 14: 5 \%, 6: 5 \%$; (d) (i) TEMPO, IBDA, $\mathrm{CH}_{2} \mathrm{Cl}_{2}$, r.t., $2 \mathrm{~h}$ (ii) $\mathrm{Et}_{3} \mathrm{~N} 3 \mathrm{HF}$, THF, r.t., $0.5 \mathrm{~h}$, two steps, $47 \%$; (e) (i) $\mathrm{Ac}_{2} \mathrm{O}, \mathrm{Et}_{3} \mathrm{~N}$, DMAP, $\mathrm{CH}_{2} \mathrm{Cl}_{2}, 0{ }^{\circ} \mathrm{C}, 1 \mathrm{~h}$ (ii) $\mathrm{Et}_{3} \mathrm{~N} 3 \mathrm{HF}$, THF, r.t., $0.5 \mathrm{~h}$, 2: two steps, $90 \%$, 4: two steps, $84 \%$. 


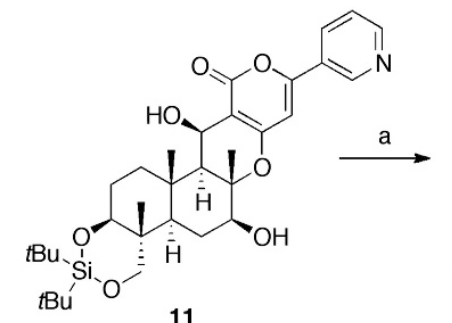

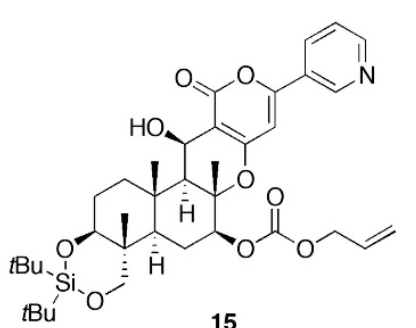

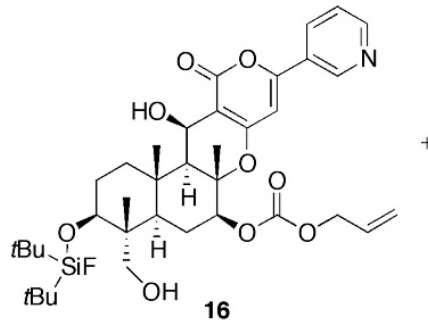

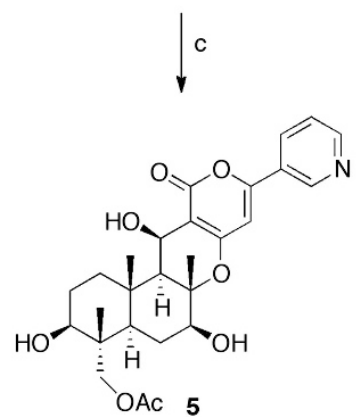
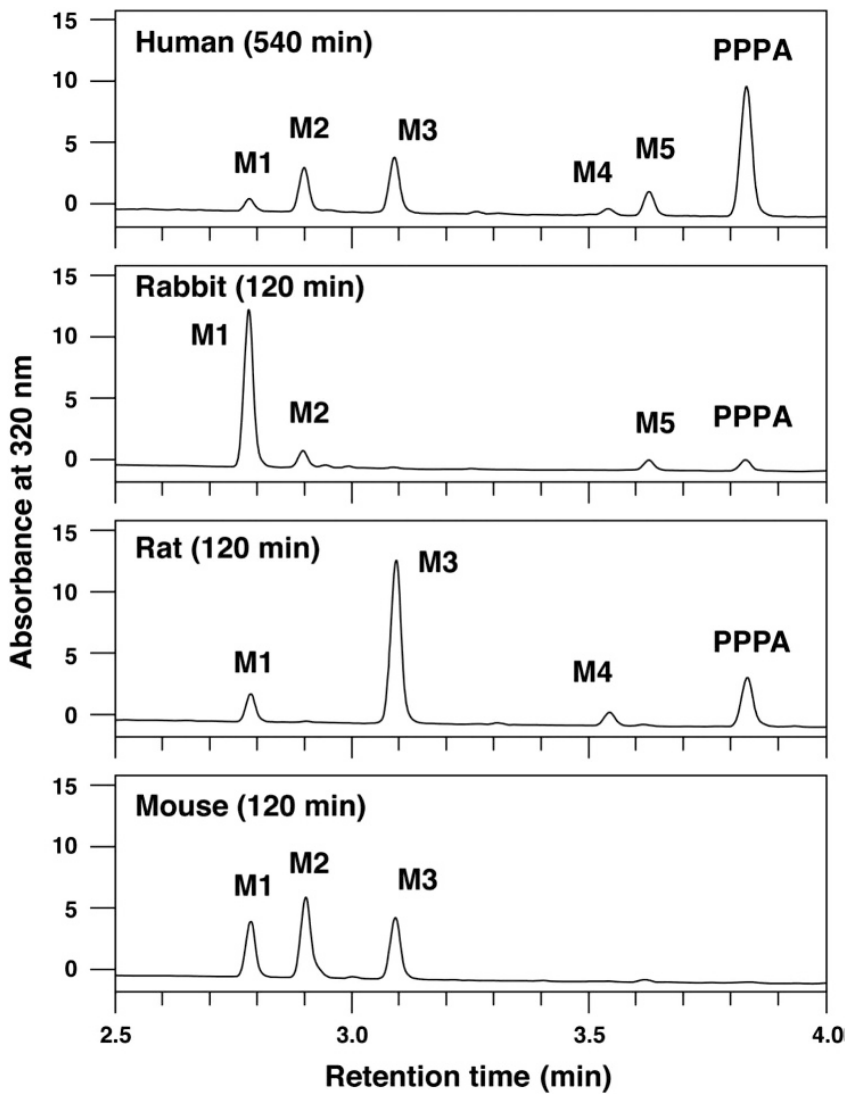

Figure 2 Representative ultra fast liquid chromatogram after incubation of pyripyropene A (PPPA) (1) in human, rabbit, rat and mouse-liver microsomes. PPPA (1) was incubated for $0,30,120,360$ or $540 \mathrm{~min}$ in the presence of human, rabbit, rat or mouse liver microsomes. The metabolites were extracted with ethyl acetate and analyzed by ultra fast liquid chromatography under the following conditions: column, Shim Pack XR-ODS (Shimadzu), $2.0 \times 75 \mathrm{~mm}$; solvent, 6-min linear gradient from $5 \%$ acetonitrile in $0.1 \%$ phosphoric acid to $95 \%$ acetonitrile in $0.1 \%$ phosphoric acid; flow rate, $0.55 \mathrm{ml} \mathrm{min}^{-1}$; detection, UV radiation at $320 \mathrm{~nm}$.

metabolites were observed (data not shown). With liver microsomes, five metabolites $(\mathrm{M} 1 \sim \mathrm{M} 5)$ were observed from human in UFLC analysis, three metabolites (M1, M2 and M5) were from rabbit, three metabolites (M1, M3 and M4) were from rat and three metabolites (M1, M2 and M3) were from mouse (Figure 2). As shown in Table 1, the retention times of M1, M3, M4 and M5 were found to be 2.8, 3.1, 3.5 and $3.6 \mathrm{~min}$, respectively, suggesting that they were 1,11-dideacetyl PPPA (6), 1-deacetyl PPPA (2), 11-deacetyl PPPA (4) and N-oxidized PPPA (10), respectively. Furthermore, their $[\mathrm{M}+\mathrm{H}]^{+}$were detected as $500,542,542$ and 600 by LC-MS, confirming the structure as expected. However, the structure of M2 was not defined.

PPPA (1) was rapidly metabolized to M1 and M2 in mouse-liver microsomes, and to M1 in rat and rabbit liver microsomes, while slowly to M3 in human-liver microsomes. Furthermore, the half life times of 1 were found to be 520,55, 72 and 15 min in human, rabbit, rat and mouse-liver microsomes, respectively (Figure 3 ).

Next, the structure of M2 was elucidated as follows: M2 was purified with high-performance liquid chromatography (column, YMC-Pack, $6 \times 250 \mathrm{~mm}$ (YMC, Kyoto, Japan); solvent, a 20-min gradient from $20 \%$ acetonitrile to $40 \%$ acetonitrile containing $0.05 \%$ trifluoroacetic acid; flow rate, $8.0 \mathrm{ml} \mathrm{min}^{-1}$; detection, UV radiation 
at $210 \mathrm{~nm}$ ), and analyzed by the linked-scan mode of fast atom bombardment mass spectroscopy (FAB-MS: JMS-DX300 (JEOL, Tokyo, Japan); Matrix: thioglycerol). As shown in Figure 4, the fragment ion peaks were observed at $m / z 79,91,104,148,202,218$, 258, 384, 392, 454 and 468 in FAB-MS, suggesting that M2 was 1-deacetyl-11-deacetoxymethyl-11-formyl PPPA (9). Furthermore, the proposed derivative 9 was synthesized, confirming that all the UFLC and LC-MS data of M2 were identical with those of 9 (Table 1). Thus, M2 was elucidated to be 9 .

In vitro PPPA metabolism in human, rabbit, rat and mouse plasma PPPA (1) was incubated with human, rabbit, rat and mouse plasma for various incubation periods. PPPA (1) was stable in human, rabbit and rat plasma at least within 540 min (Figure 5), while 1 was slowly
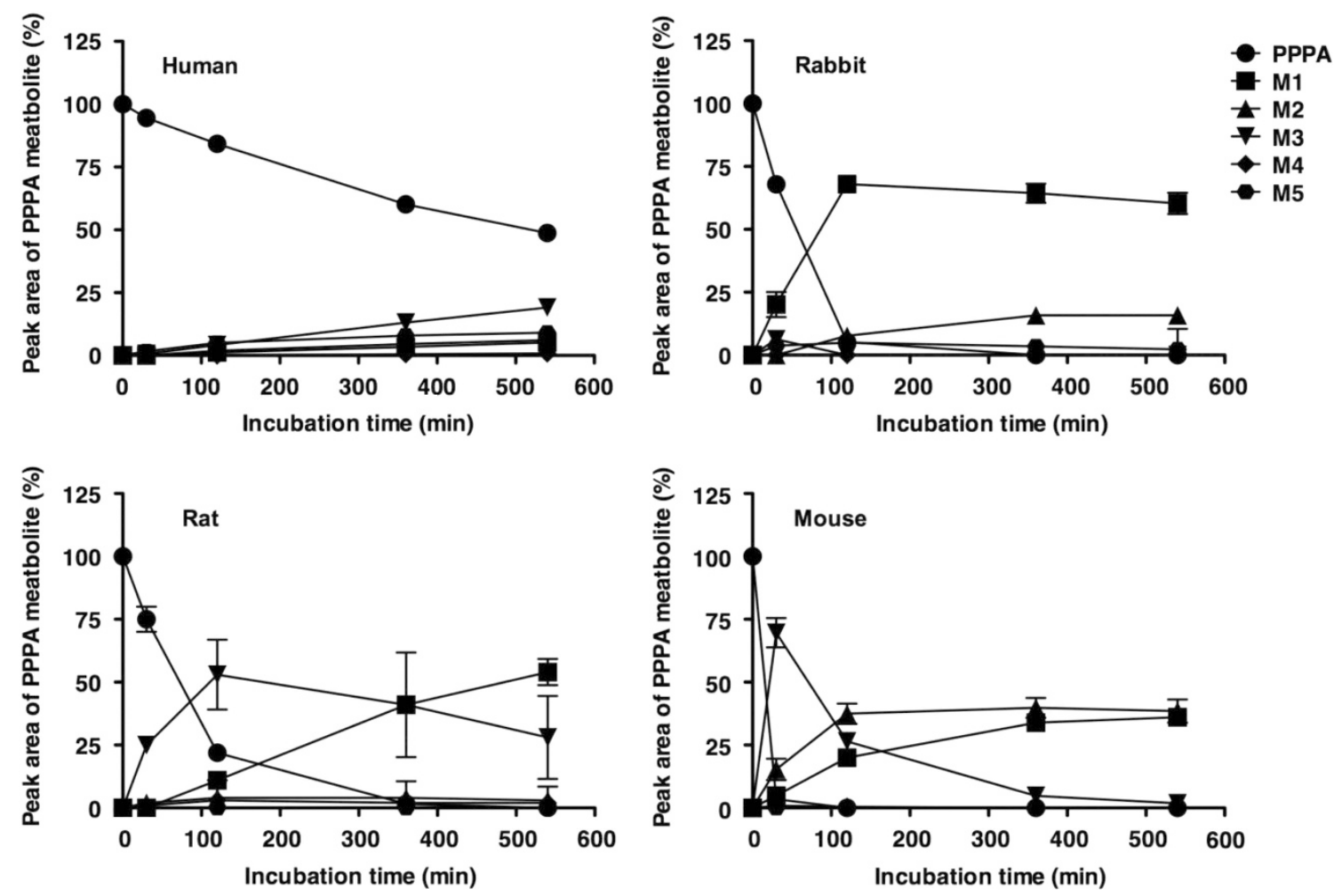

Figure 3 Metabolism of pyripyropene A (PPPA) (1) in human-, rabbit-, rat- and mouse-liver microsomes. PPPA (1) was incubated in liver microsomes at $37^{\circ} \mathrm{C}$, and production of 1 and M1 to M5 were measured and calculated as peak area of PPPA metabolite (\%). The initial peak area of 1 was defined as $100 \%$. Each value represents the mean \pm s.e.m. of more than three independent experiments.

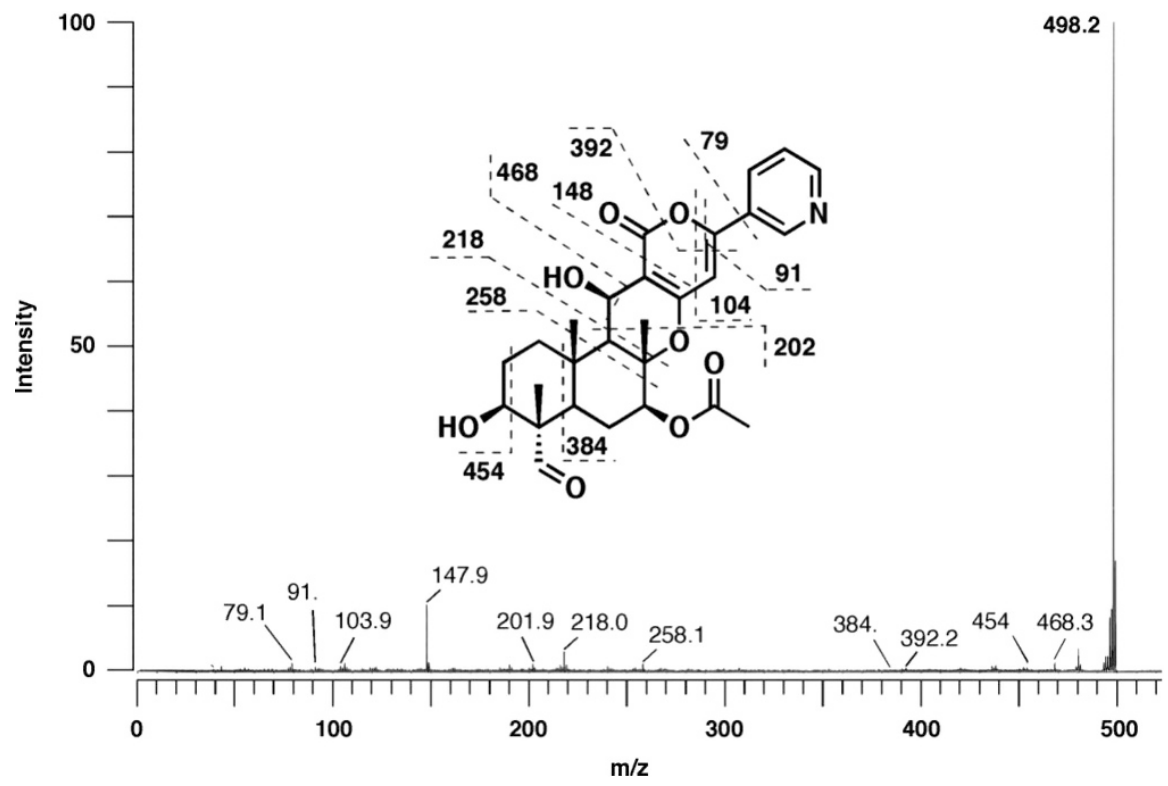

Figure 4 Mass fragments analysis of pyripyropene A metabolite M2. 

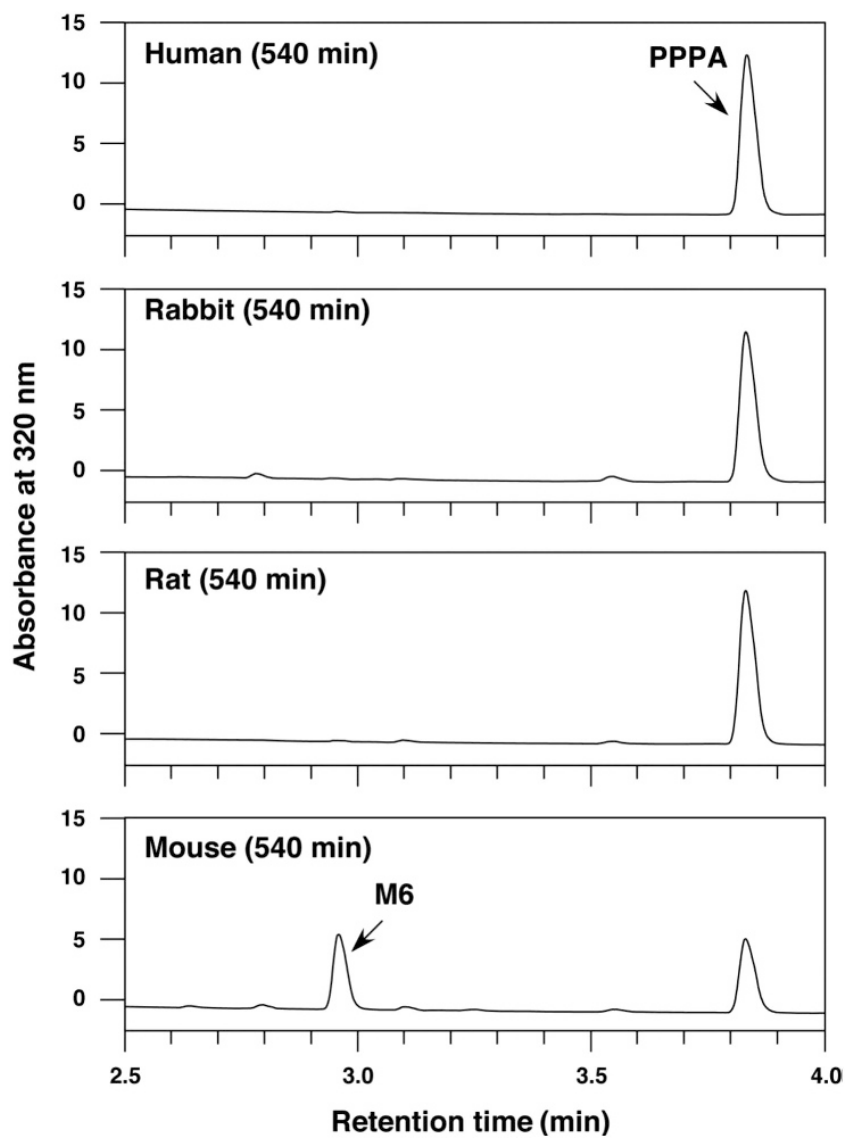

Figure 5 Representative ultra fast liquid chromatogram after incubation of pyripyropene A (PPPA) (1) in human, rabbit, rat and mouse plasmas. PPPA (1) was incubated for $0,30,120,360$ or $540 \mathrm{~min}$ in the presence of human, rabbit, rat or mouse plasma. The metabolites were extracted with ethyl acetate and analyzed by ultra fast liquid chromatography under the following conditions: column, Shim Pack XR-ODS (Shimadzu), $2.0 \times 75 \mathrm{~mm}$; solvent, 6 -min linear gradient from $5 \%$ acetonitrile in $0.1 \%$ phosphoric acid to $95 \%$ acetonitrile in $0.1 \%$ phosphoric acid; flow rate, $0.55 \mathrm{ml} \mathrm{min}^{-1}$; detection, UV radiation at $320 \mathrm{~nm}$.

metabolized to M6 in mouse plasma. M6 was eluted at $3.0 \mathrm{~min}$ by UFLC and at $7.4 \mathrm{~min}$ with $\mathrm{m} / z[\mathrm{M}+\mathrm{H}]^{+}$of 542 by LC-MS (Table 1), indicating that M6 is 7-deacetyl PPPA (3).

\section{Effect of PPPA metabolites on ACAT isozymes}

It is important to see whether or not PPPA metabolites inhibit ACAT isozymes. Therefore, synthetic PPPA derivatives were tested in the cell-based assay using ACAT1- and ACAT2-Chinese hamster ovary cells. As shown in Table 2, all derivatives lost or markedly decreased ACAT2 inhibitory activity as compared with 1. 1-deacetyl and/or 11-deacetyl derivatives $(2,4$ and 6$)$ still showed weak inhibition but were 170 to 500 times less potent than $\mathbf{1}$.

\section{DISCUSSION}

PPPA (1), a potent and selective inhibitor of ACAT2, recently proved orally active in atherogenic mouse models. ${ }^{25}$ Since 1 was originally discovered in 1993, new PPPA derivatives (the second generation derivatives) with more potent and more selective activity against ACAT2 than 1 were required. For this purpose, potential in vitro metabolism of 1 was investigated.
Table 2 Effect of PPPA and metabolites on ACAT1 and ACAT2 isozymes

\begin{tabular}{lccc}
\hline & \multicolumn{2}{c}{$C_{50}(\mu M)^{\mathrm{a}}$} & \\
\cline { 2 - 3 } \multicolumn{1}{l}{ PPPA derivative } & ACAT1 & ACAT2 & \multicolumn{1}{c}{$S /^{\mathrm{b}}$} \\
\hline PPPA (1) & $>100$ & 0.070 & $>1000$ \\
1-deacetyl PPPA (2) & $>92$ & 35.0 & $>2.6$ \\
7-deacetyl PPPA (3) & $>92$ & $>92$ & - \\
11-deacetyl PPPA (4) & $>92$ & 29.0 & $>3.2$ \\
1,7-dideacetyl PPPA (5) & $>95$ & 62.0 & $>1.5$ \\
1,11-dideacetyl PPPA (6) & $>100$ & 12.0 & $>8.2$ \\
7,11-dideacetyl PPPA (7) & $>95$ & $>95$ & - \\
1,7,11-trideacetyl PPPA (8) & $>110$ & $>110$ & - \\
1-deacetyl-11-deacetoxymethyl- & $>96$ & 72.0 & $>1.3$ \\
11-formyl PPPA (9) & & & \\
N-oxidized PPPA (10) & $>84$ & 41.0 & $>2.0$ \\
\hline
\end{tabular}

Abbreviations: ACAT, acyl-CoA:cholesterol acyltransferase; PPPA, pyripyropene A; SI, selectivity index.

${ }^{a} C_{50}$ was calculated from the two independent experiments.

belectivity index (SI): IC 50 for ACAT1/IC 50 for ACAT2.

From the present data, the in vitro metabolic pathway of $\mathbf{1}$ in the liver and plasma was illustrated in Scheme 3. In mouse liver (Figure 3), 1 was exclusively metabolized to M3 (2) then to M1 (6), indicating that hydrolysis of 1 occurs first at the 1-O-acetyl residue then at the 11-O-acetyl residue. The 7-O-acetyl residue was found to be resistant to hydrolytic enzymes in the mouse-liver microsomes. This ordered metabolic pathway of $\mathbf{1}$ appeared common in the liver of all species tested, although the half life times of 1 are distinct among the species; mouse liver with the shortest half life of $15 \mathrm{~min}$ and human liver with the longest one of $520 \mathrm{~min}$, indicating that the esterase activity in human is lower than that in nonhuman primates and rodents. These findings were comparable with the previous studies. ${ }^{27-29}$ Interestingly, there are minor metabolic pathways of $\mathbf{1}$ in human liver; first hydrolysis occurred at the 11-O-acetyl residue to produce 4 and $\mathrm{N}$-oxidation occurred to produce 10. In human, rat and rabbit plasma, 1 was stable, but in mouse plasma 1 was hydrolyzed at 7-O-acetyl residue.

From the in vitro metabolic study of 1 , there are two issues to be made clear. First, it was demonstrated that $\mathbf{1}$ is more stable in human than in mouse, suggesting that the doses for human use might be lower $(<25 \mathrm{mg} / \mathrm{kg} /$ day $)$ than those for mouse use (doses $>25 \mathrm{mg} / \mathrm{kg} /$ day are effective to show atheroprotective activity in mouse model ${ }^{25}$ ). Second, $\mathbf{1}$ is mainly hydrolyzed at the $1-O$-acetyl and the 11-O-acetyl residues, while the 7-O-acetyl residue is resistant. These PPPA metabolites had much lower ACAT inhibitory activity compared with 1 . Based on these findings, the second generation PPPA derivatives were semi-synthetically prepared, leading to the discovery of promising candidates, which are resistant to hydrolytic enzymes in liver microsomes. ${ }^{1,30-32}$ Furthermore, we found that certain PPPA derivatives proved more potent in atherogenic mouse and rabbit models than 1 . The detail will be published elsewhere.

In summary, we showed in vitro the main metabolic pathways of $\mathbf{1}$ in liver microsome and plasma of human, rabbit, rat and mouse. Our findings are expected to support the synthesis studies of PPPA derivatives, safety studies and clinical trials for the development of novel anti-atherosclerotic drugs. 

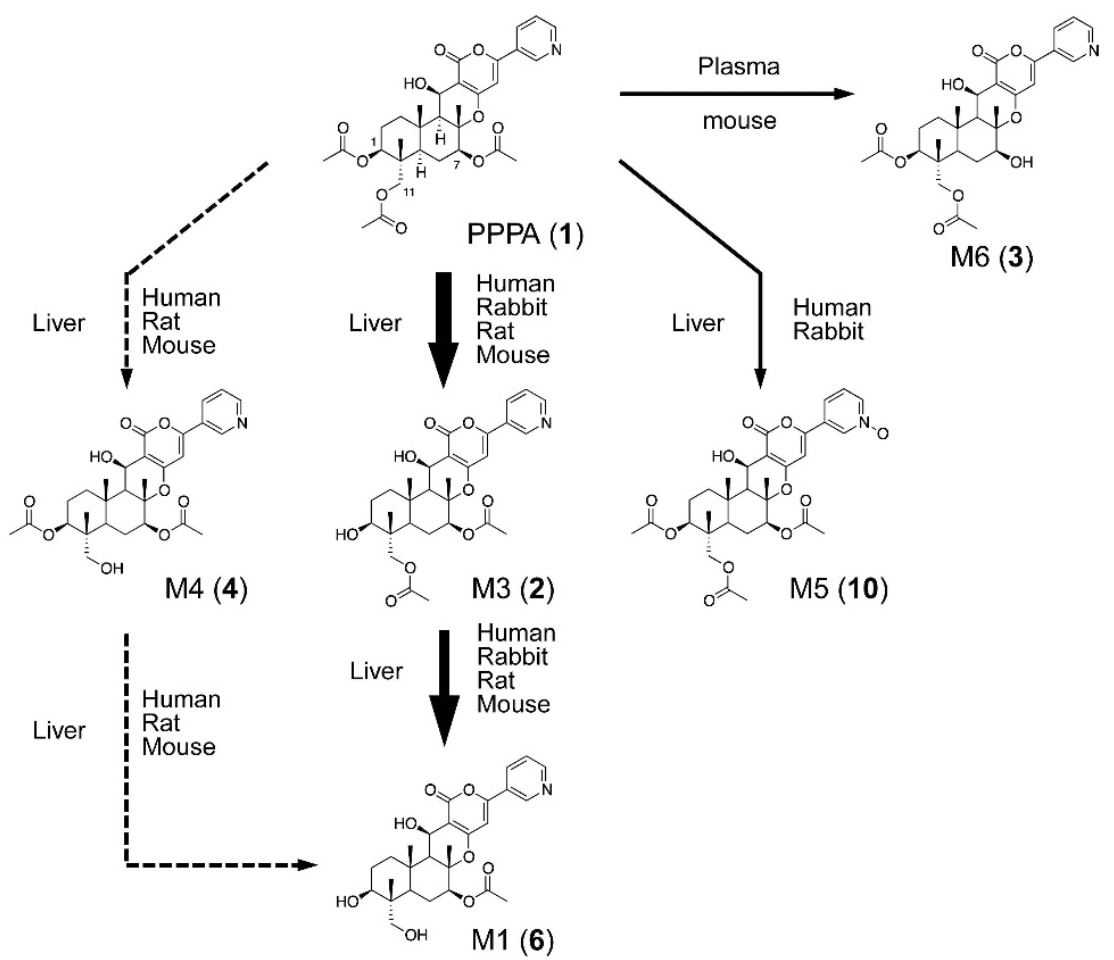

Liver Human

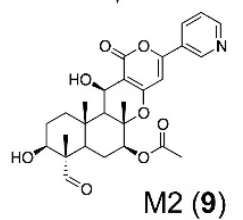

Scheme 3 Proposed metabolic pathway for pyripyropene A (PPPA) (1). The line indicated the metabolized potency: bold line > line > broken line.

\section{EXPERIMENTAL PROCEDURE}

\section{Materials}

Acetonitrile (high-performance liquid chromatography grade) and phosphoric acid were obtained from Kanto Chemicals (Tokyo, Japan). D-Glucose-6phosphate and glucose 6-phosphate dehydrogenase were from Wako Pure Chemicals (Osaka, Japan). $\left[1-{ }^{14} \mathrm{C}\right]$ Oleic acid $(1.85 \mathrm{GBq} \mathrm{mmol})$ was from PerkinElmer (Waltham, MA, USA). Ham's nutrient mixture, F-12 medium, trifluoroacetic acid and NADP were from Sigma Aldrich (St Louis, MO, USA) Penicillin (10000 units $\left.\mathrm{ml}^{-1}\right) /$ streptomycin $\left(10000 \mu \mathrm{g} \mathrm{ml}^{-1}\right)$ solution and MEM vitamins were from Invitrogen (Carlsbad, CA, USA). Geneticin was from Calbiochem (Darmstadt, Germany). Fetal bovine serum was from Hyclone (Waltham, MA, USA). Human-liver microsomes (pooled from 50 donors of mixed gender) and liver microsomes from New Zealand rabbits (male), Sprague Dawley rats (female) and CD-1 mice (female) were purchased from Xenotech (Lenexa, KS, USA). Plasma from humans (pooled from 50 donors) was obtained from George King Bio-Medical (Overland Park, KS, USA), while plasma from rabbits (New Zealand rabbit of mixed sex) and rat (Wistar rat of mixed sex) was from Rockland (Gilbertsville, PA, USA). PPPA (1) was purified from the culture broth of A. fumigatus FO-1289 according to our established method. ${ }^{15-17}$ Blood of female C57BL/6J mice (female, Japan SLC, Shizuoka, Japan) was collected in a container containing sodium EDTA and centrifuged for $10 \mathrm{~min}$ at 3000 r.p.m. at $4{ }^{\circ} \mathrm{C}$. The plasma was frozen and stored at $-80^{\circ} \mathrm{C}$. Mice were maintained and cared for according to the regulations of the Animal Care Committees of Kitasato University.

\section{Synthesis of pyripyropene derivatives}

Possible PPPA derivatives (reference standards) were synthesized according to the previously reported methods. ${ }^{21-23}$ Known PPPA derivatives 3, 8 and 10 (Table 1) were synthesized according to the previously reported methods from 1. ${ }^{21-23}$ The other novel PPPA derivatives 2, 4, 5, 6, 7 and 9 (Table 1) were prepared on the basis of the synthetic strategy developed by our group. ${ }^{31,33}$ ${ }^{1} \mathrm{H}$ - and ${ }^{13} \mathrm{C}-\mathrm{NMR}$ spectra were obtained on VARIAN MERCURY plus-300 and XL-400 (Varian Inc., Palo Alto, CA, USA), and chemical shifts were reported on the $\delta$ scale and referenced to tetramethylsilane. Mass spectra were measured with JMS-AX505HA spectrometers (JEOL, Tokyo, Japan). Unless otherwise indicated, commercial reagents were used without further purification. Organic solvents were distilled and dried over molecular sieves ( 3 or $4 \AA$ ) prior to use. Reactions were carried out in flame-dried glassware under a positive pressure of argon with magnetic stirring, unless otherwise indicated. Flash column chromatography was carried out on silica gel $60 \mathrm{~N}$ (spherical, neutral, particle size: $40-50 \mu \mathrm{m}$ ). Thin layer chromatography was performed on 0.25-mm silica gel $60 \mathrm{~F}_{254}$ plates (Merck, Darmstadt, Germany). Plates were visualized by UV radiation $(254 \mathrm{~nm})$ and anisaldehyde staining. The detail of these syntheses is described below.

\section{Assay for in vitro metabolism in liver microsomes or plasma}

Experiments of in vitro metabolism of $\mathbf{1}$ in liver microsomes and plasma were carried out according to the established method ${ }^{34}$ with minor modifications. Microsomes $\left(0.20 \mathrm{pmol} \mathrm{P} 450 \mathrm{ml}^{-1}\right)$ or plasma $(10 \%(\mathrm{v} / \mathrm{v}))$ were incubated with $1\left(6.0 \mu \mathrm{g} \mathrm{ml}^{-1}\right.$ in the incubation buffer containing $100 \mathrm{~mm}$ sodium 
phosphate buffer, pH 7.4, $1.0 \mathrm{~mm}$ EDTA, $5.0 \mathrm{~mm}$ D-glucose-6-phosphate,

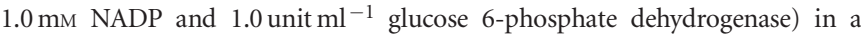
total volume of $200 \mu \mathrm{l}$ at $37^{\circ} \mathrm{C}$. The incubation was terminated by adding ethyl acetate $(500 \mu \mathrm{l})$ and distilled water $(300 \mu \mathrm{l})$, which were mixed well. After centrifugation to separate the ethyl acetate and water layers, ethyl acetate layer $(300 \mu \mathrm{l})$ was evaporated to dryness in vacuo.

\section{UFLC analysis}

For quantitative analysis of PPPA metabolites, the samples dissolved in methanol were analyzed by UFLC (Prominence, Shimadzu, Kyoto, Japan) under the following conditions: column, Shim Pack XR-ODS (Shimadzu), $2.0 \times 75 \mathrm{~mm}$; column temperature, $50^{\circ} \mathrm{C}$; solvent, 6 -min linear gradient from $5.0 \%$ acetonitrile in $0.10 \%$ phosphoric acid to $95 \%$ acetonitrile in $0.10 \%$ phosphoric acid; flow rate, $0.55 \mathrm{ml} \mathrm{min}^{-1}$; detection, UV radiation at $320 \mathrm{~nm}$.

\section{LC-MS analysis}

For structural information of PPPA metabolites, the samples dissolved in methanol were analyzed by an LC-MS system. PPPA metabolites were separated by high-performance liquid chromatography (Agilent 1200 Series LC system) under the following condition: column, Pegasil ODS (Senshu Sci. Corp., Tokyo, Japan), $2 \times 150 \mathrm{~mm}$; column temperature, $25^{\circ} \mathrm{C}$; solvent, 12 -min linear gradient from $10 \%$ acetonitrile in $0.10 \%$ formic acid to $100 \%$ acetonitrile in $0.10 \%$ formic acid; flow rate, $0.20 \mathrm{ml} \mathrm{min}^{-1}$; detection, UV radiation at $320 \mathrm{~nm}$. After each peak was separated by high-performance liquid chromatography, they were analyzed by AccuTOF time-of-flight mass spectrometer (JEOL).

\section{Assay for $\left[{ }^{14} \mathrm{C}\right]$ cholesteryl ester synthesis in ACAT1- or ACAT2- Chinese hamster ovary cells}

The assay for the synthesis of $\left[{ }^{14} \mathrm{C}\right]$ cholesteryl ester from $\left[{ }^{14} \mathrm{C}\right]$ oleic acid in ACAT1- or ACAT2-expressing Chinese hamster ovary cells ${ }^{18}$ was carried out by our established method. ${ }^{19}$ A stock solution of PPPA and its derivatives was made to a concentration of $10 \mathrm{mg} \mathrm{ml}^{-1}$ in methanol. In brief, both cell lines were maintained at $37^{\circ} \mathrm{C}$ in $5.0 \% \mathrm{CO}_{2}$ in Ham's $\mathrm{F}-12$ medium supplemented with MEM vitamins, geneticin (G418 sulfate) $\left(300 \mu \mathrm{g} \mathrm{ml}^{-1}\right)$ and $10 \%$ heatinactivated fetal bovine serum (hereafter referred to as medium A). ACAT1- or ACAT2-Chinese hamster ovary cells $\left(1.25 \times 10^{5}\right.$ cells per $0.25 \mathrm{ml}$ in medium A) were cultured in a 48-well plastic microplate and allowed to recover overnight at $37{ }^{\circ} \mathrm{C}$ in $5.0 \% \mathrm{CO}_{2}$. The assay was done with cells that were at least $80 \%$ confluent. Following the overnight recovery, a sample $(2.5 \mu \mathrm{l}$ in methanol solution) over the final concentration range of $0.0001-10 \mu \mathrm{g} \mathrm{ml}^{-1}$ with two wells for each concentration and $\left[1-{ }^{14} \mathrm{C}\right]$ oleic acid $(5.0 \mu \mathrm{l}$ in $10 \%$ ethanol/ phosphate buffered saline solution, $1.0 \mathrm{nmol}, 1.85 \mathrm{KBq}$ ) were added to each culture at $37{ }^{\circ} \mathrm{C}$ in $5.0 \% \mathrm{CO}_{2}$. After a 6-h incubation, the medium was removed, and the cells in each well were washed twice with phosphate buffered saline. The cells were lysed by adding phosphate buffered saline $(0.25 \mathrm{ml})$ containing $0.10 \%(\mathrm{w} / \mathrm{v})$ sodium dodecyl sulfate, and the cellular lipids were extracted by the method of Bligh and Dyer. ${ }^{35}$ After concentrating the organic solvent, the total lipids were separated on a TLC plate (silica gel F254, 0.5-mm thick, Merck, Darmstadt, Germany) and analyzed with a BAS2000 (Fuji Film, Tokyo, Japan). In this assay, $\left[{ }^{14} \mathrm{C}\right]$ cholesteryl ester was produced by the reaction of ACAT1 or ACAT2. ACAT inhibitory activity (\%) is defined as $\left(1-\left[{ }^{14} \mathrm{C}\right]\right.$ cholesteryl ester-drug/ $\left[{ }^{14} \mathrm{C}\right]$ cholesteryl ester-control $) \times 100$. The $\mathrm{IC}_{50}$ value is defined as the drug concentration causing 50\% inhibition of a biological activity, and is calculated from the curve fit of the data using a sigmoidal dose-response curve.

\section{ACKNOWLEDGEMENTS}

We are grateful to Prof LL Rudel (the Wake Forest University, Winston-Salem, NC, USA) for kindly providing ACAT1-CHO and ACAT2-CHO cells, and for his help in improving the manuscript. We express our thanks to Dr K Nagai for measuring mass spectra. We also thank to Ms K Fukunaga (Kitasato University) for excellent assistance. This work was supported by the Program for Promotion of Fundamental Studies in Health Science (to HT) from the National Institute of Biomedical Innovation (NIBIO).
1 Ohshiro, T. \& Tomoda, H. Isoform-specific inhibitors of ACATs: recent advances and promising developments. Future Med. Chem. 3, 2039-2061 (2011).

2 Miyazaki, A., Kanome, T. \& Watanabe, T. Inhibitors of acyl-coenzyme a: cholesterol acyltransferase. Curr. Drug Targets Cardiovasc. Haematol. Disord. 5, 463-469 (2005).

3 Rudel, L. L., Lee, R. G. \& Cockman, T. L. Acyl coenzyme A: cholesterol acyltransferase types 1 and 2: structure and function in atherosclerosis. Curr. Opin. Lipidol. 12, 121-127 (2001).

4 Dominick, M. A., Bobrowski, W. A., MacDonald, J. R. \& Gough, A. W. Morphogenesis of a zone-specific adrenocortical cytotoxicity in guinea pigs administered PD 132301-2, an inhibitor of acyl-CoA:cholesterol acyltransferase. Toxicol. Pathol. 21, 54-62 (1993).

5 Reindel, J. F., Dominick, M. A., Bocan, T. M., Gough, A. W. \& McGuire, E. J. Toxicologic effects of a novel acyl-CoA:cholesterol acyltransferase inhibitor in cynomolgus monkeys. Toxicol. Pathol. 22, 510-518 (1994)

6 Trivedi, B. K. et al. Inhibitors of acyl-CoA:cholesterol acyltransferase (ACAT). 7. Development of a series of substituted N-phenyl-N'-[(1-phenylcyclopentyl)methyl]ureas with enhanced hypocholesterolemic activity. J. Med. Chem. 37, 1652-1659 (1994).

7 Chang, C. C., Huh, H. Y., Cadigan, K. M. \& Chang, T. Y. Molecular cloning and functional expression of human acyl-coenzyme A:cholesterol acyltransferase cDNA in mutant Chinese hamster ovary cells. J. Biol. Chem. 268, 20747-20755 (1993).

8 Anderson, R. A. et al. Identification of a form of acyl-CoA:cholesterol acyltransferase specific to liver and intestine in nonhuman primates. J. Biol. Chem. 273, 26747-26754 (1998)

9 Cases, S. et al. ACAT-2, a second mammalian acyl-CoA:cholesterol acyltransferase. Its cloning, expression, and characterization. J. Biol. Chem. 273, 26755-26764 (1998).

10 Oelkers, P., Behari, A., Cromley, D., Billheimer, J. T. \& Sturley, S. L. Characterization of two human genes encoding acyl coenzyme A:cholesterol acyltransferase-related enzymes. J. Biol. Chem. 273, 26765-26771 (1998).

11 Parini, P. et al. ACAT2 is localized to hepatocytes and is the major cholesterolesterifying enzyme in human liver. Circulation 110, 2017-2023 (2004).

12 Tardif, J. C. et al. Effects of the acyl coenzyme A:cholesterol acyltransferase inhibitor avasimibe on human atherosclerotic lesions. Circulation 110, 3372-3377 (2004).

13 Nissen, S. E. et al. Effect of ACAT inhibition on the progression of coronary atherosclerosis. New Engl. J. Med. 354, 1253-1263 (2006)

14 Meuwese, M. C. et al. ACAT inhibition and progression of carotid atherosclerosis in patients with familial hypercholesterolemia: the CAPTIVATE randomized trial. JAMA 301, 1131-1139 (2009).

15 Omura, S., Tomoda, H., Kim, Y. K. \& Nishida, H. Pyripyropenes, highly potent inhibitors of acyl-CoA:cholesterol acyltransferase produced by Aspergillus fumigatus. J. Antibiot. 46, 1168-1169 (1993).

16 Tomoda, H., Kim, Y. K., Nishida, H., Masuma, R. \& Omura, S. Pyripyropenes, novel inhibitors of acyl-CoA:cholesterol acyltransferase produced by Aspergillus fumigatus. I. Production, isolation, and biological properties. J. Antibiot. 47, 148-153 (1994).

$17 \mathrm{Kim}, \mathrm{Y}$. K. et al. Pyripyropenes, novel inhibitors of acyl-CoA:cholesterol acyltransferase produced by Aspergillus fumigatus. II. Structure elucidation of pyripyropenes A, B, C and D. J. Antibiot. 47, 154-162 (1994).

18 Lada, A. T. et al. Identification of ACAT1- and ACAT2-specific inhibitors using a novel, cell-based fluorescence assay: individual ACAT uniqueness. J. Lipid Res. 45, 378-386 (2004).

19 Ohshiro, T., Rudel, L. L., Omura, S. \& Tomoda, H. Selectivity of microbial acyl-CoA: cholesterol acyltransferase inhibitors toward isozymes. J. Antibiot. 60, 43-51 (2007).

$20 \mathrm{Cho}, \mathrm{K} . \mathrm{H}$ et al. Mass-production of human ACAT-1 and ACAT-2 to screen isoformspecific inhibitor: a different substrate specificity and inhibitory regulation. Biochem. Biophys. Res. Commun. 309, 864-872 (2003).

21 Obata, R. et al. Chemical modification and structure-activity relationships of pyripyropenes. 1. Modification at the four hydroxyl groups. J. Antibiot. 49, 1133-1148 (1996).

22 Obata, R. et al. Chemical modification and structure-activity relationships of pyripyropenes. 2. 1,11-Cyclic analogs. J. Antibiot. 49, 1149-1156 (1996).

23 Obata, R. et al. Chemical modification and structure-activity relationships of pyripyropenes. 3. Synthetic conversion of pyridine-pyrone moiety. J. Antibiot. 50, 229-236 (1997).

24 Ohshiro, T. et al. Selectivity of pyripyropene derivatives in inhibition toward acylCoA:cholesterol acyltransferase 2 isozyme. J. Antibiot. 61, 503-508 (2008).

25 Ohshiro, T. et al. Pyripyropene A, an acyl-coenzyme A:cholesterol acyltransferase 2 selective inhibitor, attenuates hypercholesterolemia and atherosclerosis in murine models of hyperlipidemia. Arterioscler. Thromb. Vasc. Biol. 31, 1108-1115 (2011).

26 Imai, T., Taketani, M., Shii, M., Hosokawa, M. \& Chiba, K. Substrate specificity of carboxylesterase isozymes and their contribution to hydrolase activity in human liver and small intestine. Drug Metab. Dispos. 34, 1734-1741 (2006).

27 McCracken, N. W., Blain, P. G. \& Williams, F. M. Human xenobiotic metabolizing esterases in liver and blood. Biochem. Pharmacol. 46, 1125-1129 (1993).

28 Taketani, M., Shii, M., Ohura, K., Ninomiya, S. \& Imai, T. Carboxylesterase in the liver and small intestine of experimental animals and human. Life Sci. 81, 924-932 (2007).

29 Berry, L. M., Wollenberg, L. \& Zhao, Z. Esterase activities in the blood, liver and intestine of several preclinical species and humans. Drug Metab. Lett. 3, 70-77 (2009).

30 Ohtawa, M. et al. Synthesis and structure-activity relationship of pyripyropene A derivatives as potent and selective acyl-CoA:cholesterol acyltransferase 2 (ACAT2) inhibitors: Part 1. Bioorg. Med. Chem. Lett. 23, 1285-1287 (2013). 
31 Ohtawa, M. et al. Synthesis and structure-activity relationship of pyripyropene A derivatives as potent and selective acyl-CoA:cholesterol acyltransferase 2 (ACAT2) inhibitors: part 2. Bioorg. Med. Chem. Lett. 23, 2659-2662 (2013).

32 Ohtawa, M. et al. Synthesis and structure-activity relationship of pyripyropene A derivatives as potent and selective acyl-CoA:cholesterol acyltransferase 2 (ACAT2) inhibitors: Part 3. Bioorg. Med. Chem. Lett. 23, 3798-3801 (2013).
33 Ohtawa, M., Tomoda, H. \& Nagamitsu, T. Regioselective mono-deprotection of di-tertbutylsilylene acetal derived from 1,3-Diol with ammonium fluoride. Bull. Chem. Soc. Jpn 87, 113-118 (2014).

34 Holleran, J. L. et al. In vitro metabolism of the phosphatidylinositol 3-kinase inhibitor wortmannin, by carbonyl reductase. Drug Metab. Dispos. 32, 490-496 (2004).

35 Bligh, E. G. \& Dyer, W. J. A rapid method of total lipid extraction and purification. Can. J. Biochem. Physiol. 37, 911-917 (1959). 